(CASE REPORT)

\title{
Cortical Laminar necrosis in a patient with chronic cerebral infarction; Case report
}

\author{
Moustafa EM Radwan 1,2,* \\ ${ }^{1}$ Department of Radiology, Taibah University, Almadinah Almunawarah, Saudi Arabia \\ 2 Department of Radiology, Assiut University, Egypt.
}

Publication history: Received on 31 October 2020; revised on 09 November 2020; accepted on 11 November 2020

Article DOI: https://doi.org/10.30574/wjarr.2020.8.2.0407

\begin{abstract}
Cortical laminar necrosis (CLN) is a persistent ischemic injury attributed to a particular pan necrosis of the cerebral cortex (comprising neurons, glia, and blood vessels although underline white matter is totally or partially spared). CLN is represented radiologically by the typical curvilinear gyriform distribution high signal intensity cortical lesions on T1 weighted MRI images in the affected cerebral convolutions. This is a case of cortical laminar necrosis following old left temporo-parietal ischemic infarction. A 67-year male patient with a prior history of old left temporo-parietal ischemic infarction came for follow up MRI for old right-sided hemiplegia and aphasia. He was diabetic and hypertensive. MRI Brain images showed large old left temporo-parietal ischemic infarction in the territory of Lt. MCA. There is associated subacute ischemic infarct at the left occipital cortex. There is laminar linear cortical hyperintensity in T1WI following gyral distribution, accompanied by loss of the volume of the underlying cortex at the left temporo-parieto-occipital region suggesting cortical laminar necrosis and this picture appeared two months following old cerebral infarction and shortly the patient died.
\end{abstract}

Keywords: Cortical Laminar necrosis; Magnetic resonance Imaging; Cerebral infarction.

\section{Introduction}

Cortical laminar necrosis (CLN) is a persistent ischemic injury attributed to a particular pan necrosis of the cerebral cortex (comprising neurons, glia, and blood vessels although underline white matter is totally or partially spared)[1]. The gray matter of the cerebral cortex consists of six layers [2]. The third layer is the most able to be harmed, then comes the fifth or sixth, with the second and fourth being approximately less susceptible to ischemic necrosis from hypoperfusion[1].

CLN mostly occurs when the degree of hypoxic injury is not enough to cause total cortical damage. The white matter is usually not affected in the ischemic manifestation as it is less metabolically active [3]. It believed to be attributed to hypoxic encephalopathy, hypoglycemic encephalopathy, immunosuppressive therapy, status epilepticus, and cerebral infarction [4]. CLN characterized by neuronal ischemia associated with gliosis and the deposition of fat-filled loaded macrophages layers [5].

CLN is represented radiologically by the typical curvilinear gyriform distribution high signal intensity cortical lesions on T1 weighted MRI (T1WI) images in the affected cerebral convolutions [6]. This laminar pattern is thought to be due to the higher sensitivity of the gray matter than the white matter to the effect of hypoperfusion [7].

\footnotetext{
* Corresponding author: Moustafa EM Radwan

Department of Radiology, Taibah University, Taibah University, Almadinah Almunawarah, Saudi Arabia. 


\section{Case report}

A 67-year male patient with a prior history of old left temporo-parietal ischemic infarction came for follow up MRI for old right-sided hemiplegia and aphasia. He was diabetic and hypertensive. The patient had a history of developing the symptoms during doing coronary angiography. Follow up Magnetic resonance Imaging (MRI) of the brain was done after two months of the first attack due to deterioration of symptoms.

MRI Brain images [Figure 1; (a-k)] showed large old left temporo-parietal- ischemic infarction in the territory of Lt. MCA, that showed hypointense signal in both diffusion weighted sequence (DWI) and T1WI, with hyperintense signal in T2 weighted MRI (T2WI) and FLAIR sequences. There is associated area of subcortical hyperintensity at the left occipital cortex in both T2WI and FLAIR sequences and isointense signals in DWI with no restricted diffusion and showed faint hypointense signal in T1WI suggesting subacute insult. There is laminar linear cortical hyperintensity in T1WI following gyral distribution, accompanied by loss of the volume of the underlying cortex at the left temporoparieto-occipital region suggesting cortical laminar necrosis. Magnetic Resonance Angiography (MRA) detected occlusion at M2 segment of left middle cerebral artery (MCA) with attenuated peripheral branches and shortly the patient died.

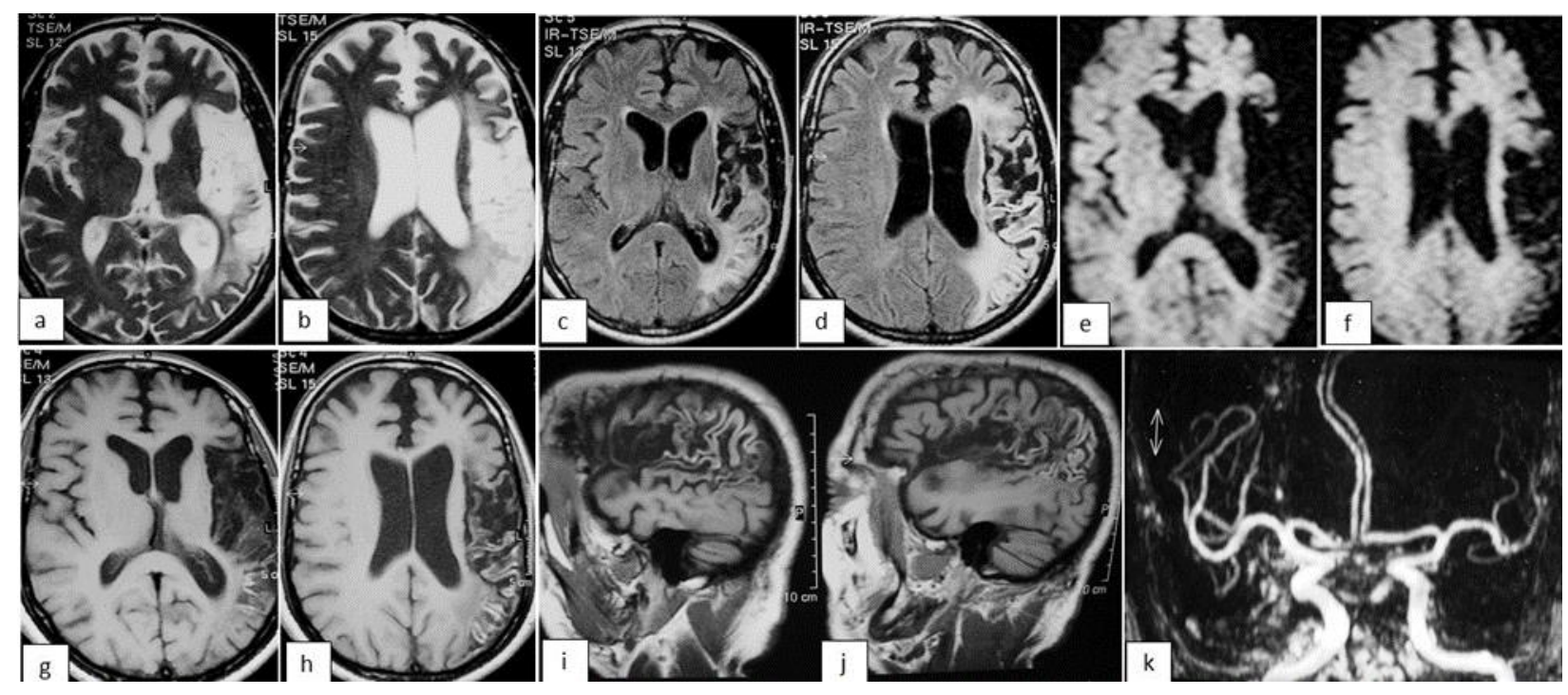

Figure 1 MRI brain of a case of cortical laminar necrosis (a-k); (a\&b) Axial T2WI, (c\&d) Axial FLAIR, (e\&f) Axial DWI, (g\&h) Axial T1WI, (i\&j) Sagittal T1WI and (k) 3D TOF MRA.

\section{Discussion}

Cortical laminar necrosis is considered a specific type of infarction that affects the cerebral cortex, which for the most part creates following summed up hypoxia instead of an abnormality within the local vasculature. Deficiency of oxygen or glucose as in anoxia, hypoglycemia, status epilepticus, ischemic cerebral stroke and more uncommon in immunosuppressive treatment and polychemotherapy has been assigned as a leading cause of cortical laminar necrosis [8].

The patient introduced in this report is consistent with patients at risk of vascular events, senior patients, male $>$ female, history of vascular disease risk factors (diabetes and hypertension) and the old cerebral infarct was developed during doing coronary angiography [9].

This case introduced CLN in a patient with old cerebral ischemic infarction. MRI is useful for detection and characterization of brain infarcts, yet a few MRI reports have documented the presence of CLN caused by ischemic stroke [10]. The CLN, seen as a laminar high signal lesion on T1WI, was first discussed by Sawada et al., in a patient with anoxic encephalopathy [11].

In the presented images; there is laminar linear cortical hyperintensity in T1WI images following gyral distribution, accompanied by loss of the volume of the underlying cortex at the left temporo-parieto-occipital region suggesting cortical laminar necrosis and this picture appeared two months following old cerebral infarction. This matched the 
results of previous studies that mentioned "the typical hyperintense cortical signal lesion on T1WI usually appears only 2 weeks after the clinical onset during the subacute or chronic phases" $[1,6]$. Other studies mentioned that CLN usually shows characteristic cortical high intensity on T1WI from 2 weeks to 2 years after ischemic insult [5, 11-13].

In spite of the fact that the mechanism of T1 shortening in CLN stays indistinct it is accepted that the T1WI hyperintensity saw in CLN is believed to be because of the particular neuronal damage, trailed by higher concentration of proteins (and other macromolecules), glial cell multiplication, and deposition of fat-loaded macrophages in the cortical area affected that enhance relatively by confining the movement of water molecules, thus causing T1 shortening [14-15].

\section{Conclusion}

Cortical laminar necrosis is a specific type of cortical infarction. CLN is characterized radiologically by high intensity cortical lesions on T1WI which shows a typical curvilinear gyriform distribution, following the cerebral convolutions affected.

\section{Compliance with ethical standards}

\section{Acknowledgments}

The authors are very thankful to all the associated personnel in any reference that contributed in/for the purpose of this research.

\section{Disclosure of conflict of interest}

The author declares no conflict of interest.

\section{Statement of ethical approval}

The present research work does not contain any studies performed on animals/humans' subjects.

\section{Statement of informed consent}

Informed consent was obtained from all individual participants included in the study.

\section{References}

[1] Siskas, NA, Lefkopoulos I, Ioannidis A, Charitandi AS. Dimitriadis. Cortical laminar necrosis in brain infarcts: serial MRI. Neuroradiology. 2003; 45(5): 283-8.

[2] Sethi, NKJ, Torgovnick C, Macaluso E. Arsura. Cortical laminar necrosis following anoxic encephalopathy. Neurol India. 2006; 54(3): 327.

[3] Cannella RG, Sparacia, V Lo Re, E. Oddo, G. Mamone, R. Miraglia. Advanced magnetic resonance imaging of cortical laminar necrosis in patients with stroke. Neuroradiol J. 2019; 32(6): 431-437.

[4] Serrano-Pozo AJR, González-Marcos A. Gil-Peralta. [Cortical laminar necrosis caused by cerebral infarction]. Neurologia. 2006; 21(5): 258-9.

[5] Sharma SR, H Massaraf, S Das, A Kalita. Cortical Laminar Necrosis as a Presenting Manifestation of Migraine in an Apparently Normal Patient: A Rare Case Report. J Neurosci Rural Pract. 2019; 10(3): 559-562.

[6] Komiyama M, H Nakajima, M Nishikawa, T Yasui. Serial MR observation of cortical laminar necrosis caused by brain infarction. Neuroradiology. 1998; 40(12): 771-7.

[7] Morais R, F Sobral, G Cunha, O Brito, I Santana. Advanced MRI study of migrainous infarction presenting as cortical laminar necrosis - Case report and literature review. Clin Neurol Neurosurg. 2018; 167: 82-85.

[8] Samain, JL, E Haven, M Gille, P Mathys. Typical CT and MRI features of cortical laminar necrosis. Jbr-btr. 2011; 94(6): 357.

[9] Cardiovascular Disease and Risk Management. J Diabetes Care, 38(Supplement 1) 2015; S49-S57. 
[10] Nabatame H, N Fujimoto, K Nakamura, Y Imura, Y Dodo, H Fukuyama, J Kimura. High intensity areas on noncontrast T1-weighted MR images in cerebral infarction. J Comput Assist Tomogr. 1990; 14(4): 521-6.

[11] Takahashi S, S Higano, K Ishii, K Matsumoto, K Sakamoto, Y Iwasaki, M. Suzuki. Hypoxic brain damage: cortical laminar necrosis and delayed changes in white matter at sequential MR imaging. Radiology. 1993; 189(2): 44956.

[12] Allen LM, AN Hasso, J Handwerker, H. Farid. Sequence-specific MR imaging findings that are useful in dating ischemic stroke. Radiographics. 2012; 32(5): 1285-97.

[13] Komiyama M, M Nishikawa, T. Yasui. Cortical laminar necrosis in brain infarcts: chronological changes on MRI. Neuroradiology 1997; 39(7): 474-9.

[14] Arboix A, S Bechich, M Oliveres, L García-Eroles, J Massons, C Targa. Ischemic stroke of unusual cause: clinical features, etiology and outcome. Eur J Neurol. 2001; 8(2): 133-9.

[15] el Quessar A, JC Meunier, C Delmaire, G Soto Ares, JP Pruvo. [MRI imaging in cortical laminar necrosis]. J Radiol. 1990; 80(9): 913-6. 